\title{
Pulse increase at mass interaction in an energy carrier
}

\section{Vasily Ivanovich Bogdanov}

Solovyov Rybinsk State Aviation Technical University, Doctor of Engineering, 53 Pushkin Street, Rybinsk 152934, Yaroslavl region, Russia

\section{Email address:}

bogdanov-vasiliy@yandex.ru(V. I. Bogdanov)

\section{To cite this article:}

Vasily Ivanovich Bogdanov. Pulse Increase at Mass Interaction in an Energy Carrier. Computational Biology and Bioinformatics. Vol. 2, No. 4, 2013, pp. 195-201. doi: 10.11648/j.ajmp.20130204.13

\begin{abstract}
Capability of pulse increase due to the effect of mass addition during pulsejet engines operation is shown. Under definite conditions it is possible to create a pulse even with no reaction mass ejection, which corresponds to a local solution of the Meshchersky equation according to which for a variable-mass body the expelled mass is equal to the added mass. The effect of pulse increase due to mass interaction can manifest itself in liquids, elastic solids and even in living organisms. This can explain an increased efficiency of the wing in the pulsating flow, movement of the so-called 'inerzoids' without their interaction with the environment. Possible ways of the effect realization in various spheres of human activities are considered.
\end{abstract}

Keywords: Pulsating Working Process, Added Mass, Cavity, Ejection Channel, Inerzoid, Pulsating Wing

\section{Introduction}

Nowadays intense interest is shown to application of a pulsating working process in the propulsive devices, primarily due to the capability of thermodynamic efficiency increase and simplification of the units design. However, the pulsating working process is interesting not only in terms of the thermodynamic efficiency. As can be seen from the latest investigations this process opens new opportunities for thrust momentum increase due to mass interaction.

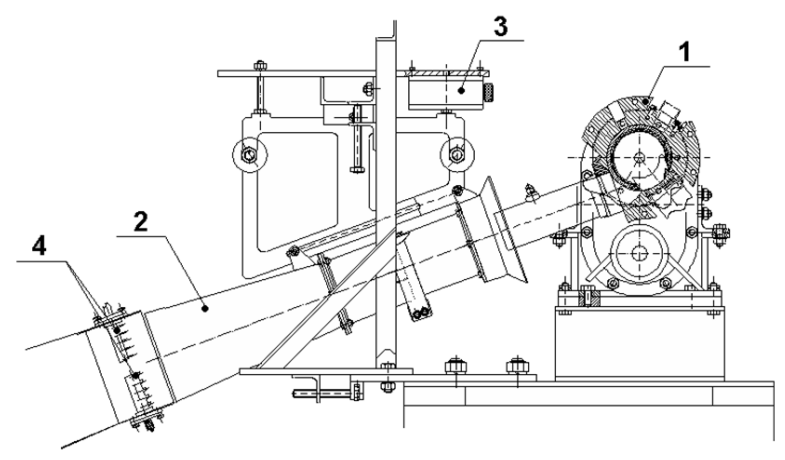

Figure 1. Pulsejet engine with a slide valve combustor and thrust augmenting ejector: 1 -pulsejet engine; 2 - ejector channel; 3 -thrust measuring gage; 4 - gas total pressure and temperature sensors.

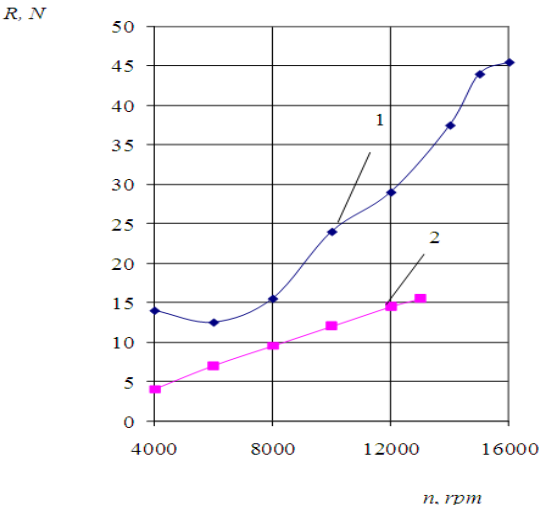

Figure 2. Pulsejet engine thrust versus slide valve rotational speed: 1 experiment; 2 -design.

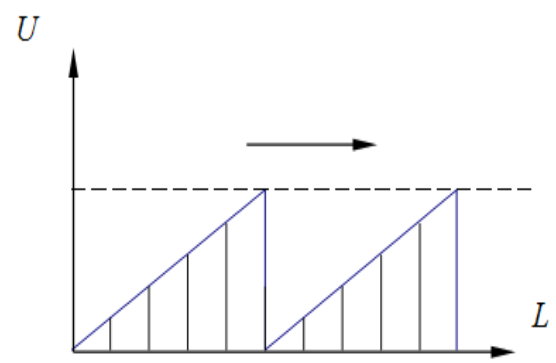

Figure 3. Typical distribution of gas cyclic mass velocity $U$ to length $L$. 


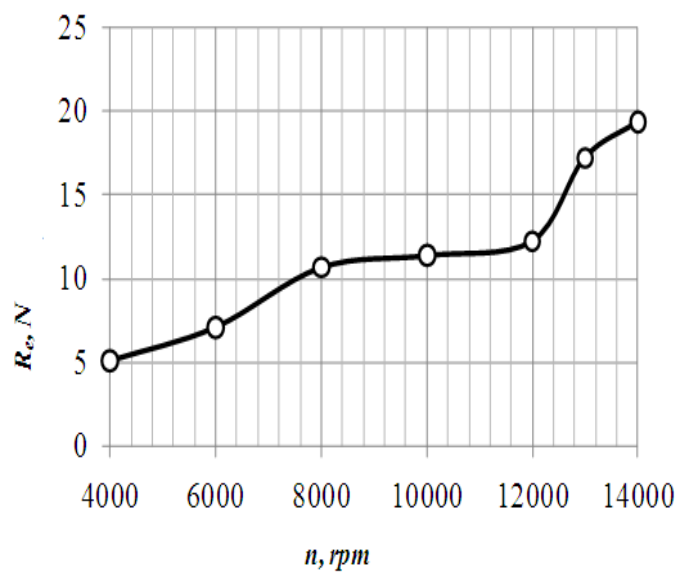

Figure 4. Forces measured on the ejector channel versus slide valve rotational speed.

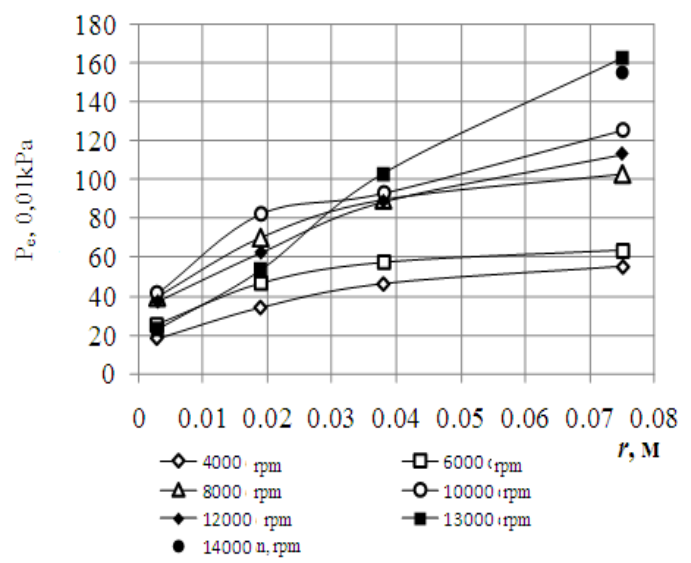

Figure 5. Distribution of the radial dynamic pressure measured at the ejector channel exit.

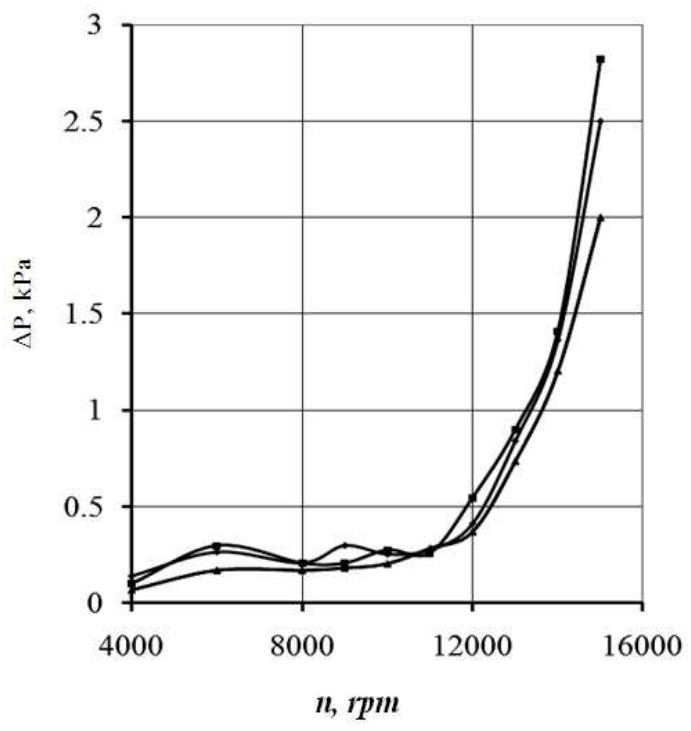

Figure 6. Pressure oscillations at the ejector channel exit.

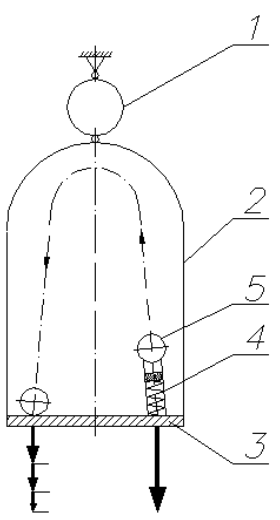

Figure 7. The design to demonstrate the pulse generation capability with no reaction mass ejection: 1 -dynamometer; 2 -vessel; 3 -thrust wall; 4 - spring; 5 - ball-cyclic mass

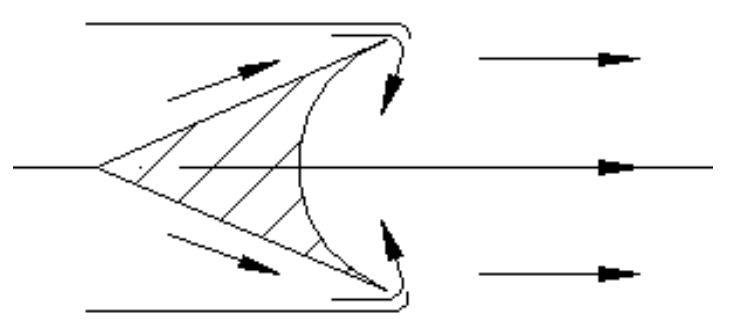

Figure 8. Design of the unit with a spherical cavity.

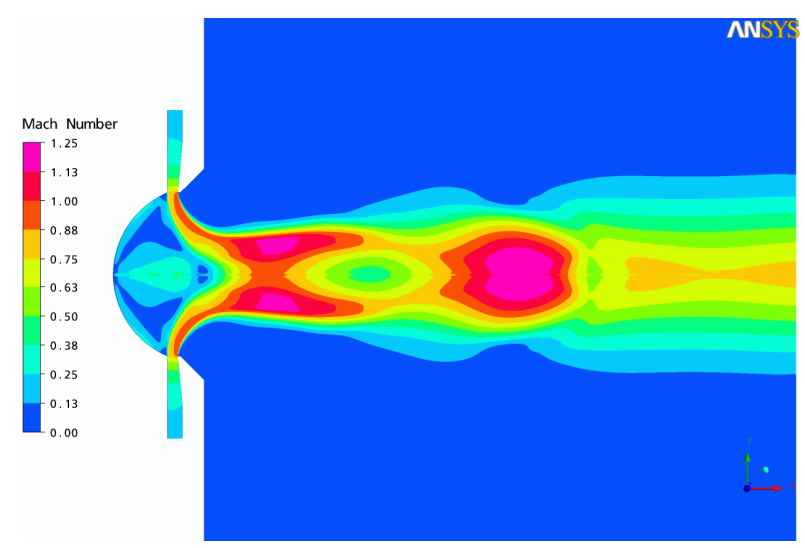

Figure 9. Flow visualization in the cavity.

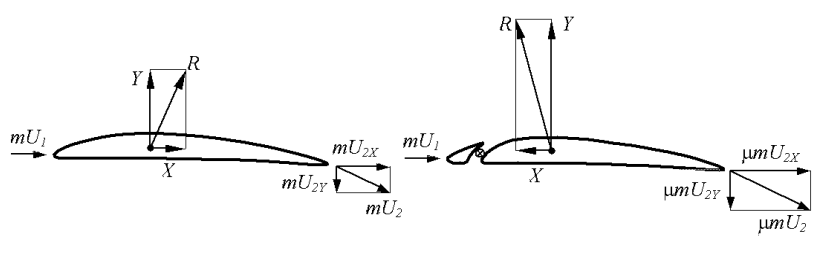

a

b

Figure 10. Interaction of the wing with steady-state (a) and pulsating (b) flows, irrespective of the hydraulic losses. 


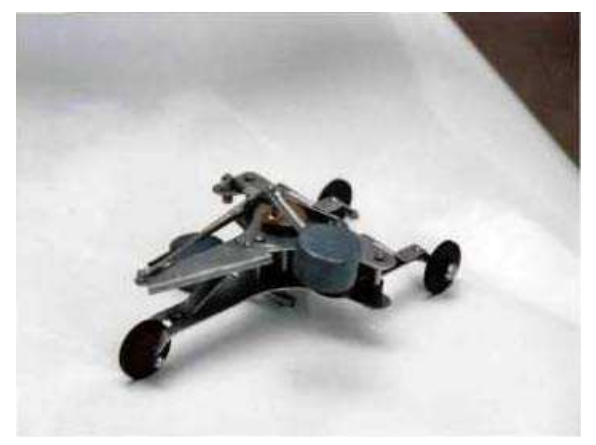

Figure 11. Inerzoid.

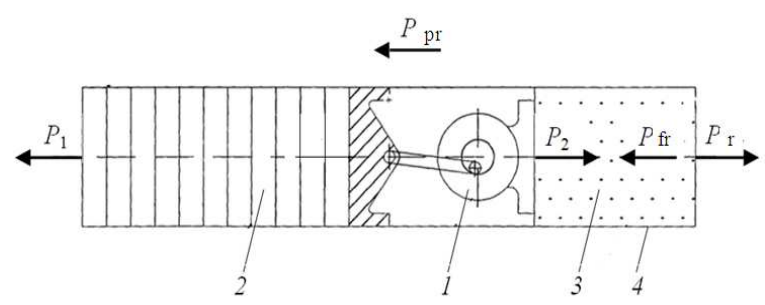

Figure 12. Diagram of propulsive device (inerzoid) with no reaction mass ejection: 1 - shock mechanism; 2 - perfectly elastic body; 3 - non-elastic body; 4 - casing.

NPO Saturn has conducted tests of the experimental pulsejet engine with the thrust-augmenting ejector based on a high-frequency slide valve constant volume ( $\mathrm{V}=$ const) combustion chamber of a new type (Fig. 1) [1, 2, 3]. The results of the thrust measurement and its estimation (based on the internal engine parameters) under assumption of quasi-steadiness of the flow process showed that measured thrust $P$ exceeds almost twice the design value (depending on slide valve rotational frequency $n$ ) (Fig. 2). This can be explained by the fact that the pulsejet engine with the slidevalve $\mathrm{V}=$ const combustion chamber has pulse duration of operational fluctuations of about $75 \%$ and in the intervals between gas jet feeds the space behind the nozzle is filled with the ambient (atmospheric) air, which with gas escape becomes an additional mass that increases the engine thrust. This proves the known design-theoretical investigation of a single cycle (one-dimensional expansion of detonation products - gas) [4] that shows the possibility of 3 times pulse increase in the atmosphere compared to the vacuum. It is also shown that with gas interaction with the atmosphere the oscillating process takes place, at the definite moments of which gas goes backward to the source. This gas can become an additional mass for the next cycle. With pulse duration of operational fluctuations close to zero, it is possible to use a portion of the cyclic mass of the used gas jet (its 'tail' having a lower speed compared to its front) as an additional mass (Fig. 3).

The test results of the pulsejet engine with thrustaugmenting ejector are shown in Fig. 4 and 5 as the force measured on the thrust-augmenting ejector, dynamic pressure at the ejector channel exit versus the slide valve rotational speed.

\section{Experiment}

Variation of the parameters within the range of slide valve rotational frequency $n$ from 12000 to $13000 \mathrm{rpm}$ is of interest. With a variation of $n$ by $8.3 \%$ force $R_{\text {eexperim. }}$ on the thrust-augmenting ejector increased by $41 \%$. The velocity field at the thrust-augmenting ejector exit sharply changed, i.e. the flow speed in the near-wall zone of the channel reduced. To explain this phenomenon, estimation of the thrust variation $R_{\text {e.calc. }}$ On the thrust-augmenting ejector to the flow parameters at the exit and the analysis of the experimental and design results [3] were performed.

Table 1

\begin{tabular}{|c|c|c|}
\hline Thrust, N & $\begin{array}{l}n, \mathrm{rpm} \\
12000\end{array}$ & 13000 \\
\hline$R_{\text {e.calc. }}$ & 10,18 & 10,23 \\
\hline$R_{\text {e.experim. }}$ & 12,14 & 17,15 \\
\hline
\end{tabular}

Based on the estimation, the force on the ejector channel is significantly less than the measured one; with increase of $\mathrm{n}$ from $12000 \mathrm{rpm}$ to $13000 \mathrm{rpm}$ it should only increase by $0,5 \%$. However, the measured force in this case increased by $41 \%$. The contradiction between the design and experimental values of the force, especially at transition from $n=12000 \mathrm{rpm}$ to $\mathrm{n}=13000 \mathrm{rpm}$, can be linked up with a sharp change of the velocity field within this slide valve rotational speed range.

Sharp reduction of the flow velocity in the near-wall zone of the ejector channel can be explained by the flow separation in the channel diffuser zone. It is known [5] that boundary-layer separation is always related to formation of vortex as a result of the direct and inverse flow interaction that can occur in the oscillating process. Addition of mass that increases the thrust [1] can occur during this process, i.e. one and the same air mass can create thrust first as an active and then as an additional mass. In this situation the kinetic energy (dynamic pressure) is converted to pulse. Exactly this explains the contradiction between the measured thrust and the results of its estimation to dynamic pressure. We can suppose that at $\mathrm{n}=12000 \mathrm{rpm}$ a moderate addition of mass without flow separation takes place, and at $\mathrm{n}=13000 \mathrm{rpm}$ a more intense addition of mass with flow separation is possible, in a resonance oscillating process with repeated addition of one and the same mass.

To confirm this and to eliminate a possible addition of the outside mass, a cylindrical screen was installed at the ejector channel exit (at a distance of $10 \ldots 20 \mathrm{~mm}$ ). The tests showed [3] that dynamics of the force flow in the ejector channel did not change, and the force values with the screen and without it only slightly changed for the same fluctuation frequencies. The characteristic feature is that the dynamics of fluctuation amplitude $\Delta \mathrm{P}$ (Fig. 6) measured at the ejector channel exit with the LX-610 sensor at $\mathrm{n}>12000 \mathrm{rpm}$ is the same as with the force measured at the ejector channel. 


\section{Intellectual Experiment}

To prove the possibility of pulse increase at the expense of the repeated cyclic mass addition in the pulsating working process, we propose to visualize the following simple experiment on the unit (Fig. 7):

On dynamometer 1 vessel 2 is hooked, which is vacuumtreated to eliminate the effect of air (as an outside mass), its bottom serves as thrust wall 3 . Spring device 4 is located inside the vessel, which can throw up a ball, i.e. cyclic mass 5. At actuation of the spring device the ball is thrown up thus creating the pulse (solid arrow) as in a typical jet engine. Upon attainment of a definite height the ball, under the influence of gravity, will return, hit the thrust wall, convert through a series of bounces the stored kinetic energy to additional pulses (dashed arrows) and remain within the unit. So, owing to the gravity force being external with regard to the unit, the whole kinetic energy of the ball can be converted to a pulse (except for the shock losses). In this limiting situation, there is no mass expulsion outside the unit. Here we have both mass removal (within the unit) and its further addition due to the gravity force. That is why here the total pulse increase is compliant with a local solution of the known Meshchersky equation according to which for a variable-mass body the expelled mass is equal to the added mass.

In a pulsating gas jet, the outside force in relation to the engine will be the force of the gas cyclic mass elastic interaction (Fig. 3) that arises at a definite combination of the working process fluctuation parameters.

If there is mass interaction in a pulsating gas jet there will be shock losses, as well. The higher the gas pressure the less kinetic energy at shock is converted to internal energy and the more energy is converted to pulse [6].

The effect of mass interaction is confirmed by other investigations, as well:

1. At NASA Glenn Research Center during experimental research of a pulsejet thrust-augmenting ejector with a resonance device at inlet [7], the ejecting process efficiency of 1.15 was obtained that contradicts with the laws of conservation. The reverse gas flow at definite periods of time was observed. However, the authors could not explain this phenomenon. The contradiction can be resolved, provided one and the same air mass is used first as an active and then as an additional mass within the oscillating process.

2. At the Institute of Mechanics of the Moscow State University [8] specific thrust parameters of the pulsating process organized in the unit on the basis of the spherical cavity (Fig. 3), corrected to the working fluid flow rate are 1.52 times higher than the values obtained at quasi-steady estimation.

This was once again confirmed by similar works conducted in 2004 at A. Lyulka Scientific-and-Technical Center (NPO Saturn). Furthermore, here at definite operating modes the direct dependence of thrust increase on the air temperature at constant pressure and unchanged flow path geometry was discovered. This is attributed to the fact that with the temperature rise the elasticity of the interacting gas cyclic masses is increased (masses are less distorted), and hence shock losses are reduced. Fig. 9 shows the gas flow in the spherical cavity, which was obtained during the design investigation using contemporary numerical techniques. The gas mass interaction at the exit from the cavity is obvious.

While in operation [1] thrust was measured by a wellknown method based on the principle of gas jet active force measurement with the thrust wall. The possibility to obtain great pressure on the wall when affected by a gas pulsating jet suggested the use of this effect as a new means of the lift force creation. To study the possibility of the obtained effect implementation on flying vehicles, the analysis [9, 10] was performed to investigate the unsteady interaction of the wing with the incident flow. The most interesting analysis results are as follows:

- The may-bug paradox opened in the 1970s by Leon Bennett from the New-York University, according to which the bug will lack lift force for a flight if we apply the stationary aerodynamic laws for determining it;

- In 1922, Katzmeir proved experimentally that the drag of the wing oscillating freely in the flow is significantly less than that of the fixed wing;

- In MAI investigations of the wing with a pulsating slat were performed; the driven slat created a pulsating flow about the wing, and the wing bearing capacity was more than double increased.

However, these publications do not contain any substantiation of the increased efficiency of the wing and the air flow unsteady interaction.

\section{Discussion of Results}

The analysis of the work performed by the may-bug wings suggests that they interact in much the same manner as in the above experiment: pulsejet engine - thrust wall. Here, to realize the maximum efficiency, the optimum mutual orientation of the pulsating air flow source and the wing is required. In a similar manner, the pulsating slat 'makes' one and the same air mass in the oscillating process over the wing outline contour participate once and again in the lift force creation [10].

The analysis of the above-mentioned investigation results shows that in all the described cases the repeated use of one and the same air (gas) mass for generating a pulse takes place [11]. In accordance with the Euler law of momentum conservation, Fig. 10 shows the diagram of the wing force interaction with steady (a) and due to the vibrating slat pulsating (b) flows. For better understanding the physics of the wing and the pulsating flow interaction process, the assumption of the absence of hydraulic losses at flow about the wing and shock losses at pulsating air flow is introduced. In addition, we assume that the axial 
force vector $\mathrm{X}$ is parallel to the velocity vector $\mathrm{U} 1$. Then the Euler equation can be written for the steady flow about the wing (a):

- $\mathrm{mU} 2 \mathrm{X}-\mathrm{m} \mathrm{U} 1=-\mathrm{X}$

- $\mathrm{m} U 2 \mathrm{Y}=\mathrm{Y}$,

For pulsating flow (b):

- $\mathrm{m} \mathrm{U} 2 \mathrm{X}-\mathrm{m} \mathrm{U} 1=\mathrm{X}$

- $\mathrm{mU} 2 \mathrm{Y}=\mathrm{Y}$,

Where: $\mathrm{mU} 1$ is the momentum of air interacting with the wing in front of the leading edge;

- is the additional mass factor at pulsating flow about the wing;

- $\mathrm{m}$ U2 is the momentum of air that have interacted with the wing behind the trailing edge;

- $\mathrm{X}$ is the axial force;

- $\mathrm{Y}$ is the lift force.

The analysis of flow about the wing conditions described above allows making the following conclusions:

For steady flow about the wing, $\mathrm{X}$ determines the inductive drag of the wing;

At pulsating flow about the wing the quantity of air movement at the wing trailing edge is increased due to mass addition, as a result of which the lift force is increased, and the axial force is generated, which is directed forward and reduces the drag force (this is precisely the case pointed out in the Katzmeir experiments);

Due to the mass addition the air flow velocity behind the wing should reduce, and with the air temperature increase we can expect increase of the pulsating flow about the wing efficiency at the expense of the shock losses reduction.

The precise assessment of the effect, optimization of the pulsating gas flow parameters can be performed by means of physical-mathematical modeling with the help of contemporary numerical techniques.

The obtained results of unsteady gas-dynamic process investigations were used to explain the causes of propulsive force origination in solids (inerzoids) [6, 12] and living organisms (levitation phenomenon). It was I. Newton who established the rules for obtaining scientific knowledge [13], among which the following were used in connection with the set task:

Ontological assumption on the nature simplicity: do not allow more causes than are enough to explain visible acts of nature; this rule evolves a well-known principle of simplicity by W. Occam pointing to the necessity of searching for simple explanations;

Ontological idea of the uniformity of nature: one and the same phenomenon should as far as possible be explained by the same causes.

Since there was no visible interaction with the ambient atmosphere in experiments with the 'inerzoids' (Fig. 11), new theories (gravitational, torsional fields, etc.) were suggested to resolve contradictions with the law of momentum conservation, which did not comply with the above-mentioned rules. However, the new theories did not give well-defined explanations of the effects achieved, and so they were subjected to criticism.

The inerzoid mechanisms, in spite of their variety, can be brought to a simplified diagram (Fig. 12), where: 1 is the shock mechanism; 2 and 3 are the bodies of various elasticity located in casing 4.

Let us examine the inerzoid loading pattern (Fig. 12). For better understanding the physics of this process let us make the following assumptions:

- The mass determining the momentum is focused within the shock mechanism;

- Body 2 is perfectly elastic;

- Body 3 is non-elastic;

- There is no friction between the parts of the shock mechanism, bodies 2 and 3 and casing 4 walls;

- $\mathrm{P} 1=\mathrm{P} 2$ are the forces transmitted by the shock mechanism to body 2 and 3;

- Pfr. Is the frictional force generated at deformation in non-elastic body 3 ;

- Pr. Is the residual force transmitted by non-elastic body 3 to the right end face of casing 4 .

Owing to perfectly elastic body 2 , force $\mathrm{P} 1$ is transmitted to the left end face completely.

Let us write a momentum equation for the assumed loading pattern. We assume the direction of propulsive force Ppr. as positive.

$$
\begin{gathered}
P_{1}-P_{2}+P_{f r}-P_{r}=\frac{m d U}{d t}=P_{p r} \\
P_{p r}=\frac{m d U}{d t}=P_{f r}-P_{r}
\end{gathered}
$$

In the closed system of a real inerzoid with a successive action by the shock mechanism on bodies 2 and 3 with available $\mathrm{P}_{\text {resid. }}$ the momentum within a period will change sign, which explains inerzoid periodic displacement backward, observed with some inerzoids.

In bodies 2 and 3 during the shock mechanism operation in the oscillating process (as in the pulsating gas flow), mass addition can occur with an efficiency conditioned by the bodies elasticity. However, this requires investigations taking into account notions of the solid-state physics.

If there were the possibility to manufacture the inerzoid mechanism from absolutely solid material, then no motion would occur.

Without any doubt, when understanding the physical character of the effect, the efficiency of mass interaction in inerzoids can be increased at optimum combination of the design, materials and motion parameters.

The known phenomenon, i.e. short-time weight reduction in the human body and other living organisms under definite conditions called 'levitation', has not received a well-defined explanation so far. Let us consider this phenomenon taking into account the known rules for obtaining scientific knowledge and using the fore-cited investigation results of the propulsive force that arises in gases and solids at mass interaction. During the experiments on levitation, muscular trembling was 
observed with a human being that can be considered as a peculiar pulsating process. The analysis of the musculoskeletal system of a human being showed its similarity with the inerzoid. More powerful difference in bones and muscles elasticity, compared to an inerzoid, is obvious. Therefore, it is reasonable for biomechanics scientists to consider the causes of origination of the force reducing the human body weight as a result of the mass interaction in muscles (at their high-frequency contraction) and bones.

It is known that in oil-well drilling, creation of pulsations in the drilling fluid close to the bottom-hole area increased the penetration speed [14]. The analysis of the investigation results allows supposing that the pulse increase at the face area due to mass interaction in the drilling fluid may be one of the causes for increasing the penetration speed.

\section{Range of Effect Application}

The obtained effects can be widely used in engineering:

\subsection{In Jet Engines.}

The biggest pulse gain due to addition of the own mass in gases can be obtained in jet engines operating at low thrust efficiency:

- First-stage launch vehicle engines;

- Brake engines;

- Spacecraft attitude engines;

- Lift engines for vertical take-off-and-landing (VTOL) aircraft.

\subsection{In Lift Force Generating Units.}

The pulsating wing can be used when installed on the pulsejet engine upper surface. Here two positive effects of the pulsating working process will be combined.

A new implementation of the pulsating slat with a new gas-dynamic method of pulsation generation is also possible. In a wing with a conventional slat, at its extension at takeoff and landing a device made on the basis of the present-day knowledge in gas-dynamic control is switched on. This device controls a high air flow in the slat channel with the help of pulsating delivery of small quantities of air taken from the engine.

In order to increase the lift force, the aerodynamic interaction of specially shaped blade rings can be organized in the VTOL fan, i.e. it is suggested to replace the wing waving action (as that with the may-bug) by the rotating one realized in the engines.

\subsection{In Propulsive Devices with No Reaction Mass Ejection and In Units with Increased Pulse Effect.}

Propulsive devices with no reaction mass ejection can find their application in continuous-duty spacecraft for orbit correction, control and stabilization. These spacecraft may have enough power from solar batteries or nuclear installation, but they will always lack expendable reaction mass.

This effect can be also used to increase the drop-hammer effect for pile driving in construction, drilling equipment efficiency at the expense of the penetration speed increase.

The effect of mass interaction in musculoskeletal system of a human being can be used, for example, to increase the sporting results under condition of new training methods elaboration.

\section{References}

[1] V. I. Bogdanov, "Interaction of masses in the operating process of pulsejet engines as a means of increasing their thrust efficiency", Journal of Engineering Physics and Thermophysics (IFZh), vol. 79, 2006, pp. 85-90.

[2] V. I. Bogdanov, "On application of the pulsating detonation working process in propulsive units", Izvestia RAN (Russian Academy of Sciences), Power generation, No. 2, 2007, pp. 76-82.

[3] V. I. Bogdanov and L. I. Burakova, "Estimation of mass interaction effects in pulsejet engines based on the experimental results", P.A. Solovyov RGATA Bulletin, Rybinsk, No. 3, 2011, pp. 90-95.

[4] F. A. Baum, L. P. Orlenko, K. P. Stanyukovich, and B. I. Shekhter, Detonation physics, Nauka, M., 1975.

[5] O. S. Sergel Applied Fluid Dynamics. M.: Machinostroyeniye, 1981, p. 374.

[6] V. I. Bogdanov, "Pulse increase due to the effect of mass addition within the engine energy carrier", Polyot, No. 8 , 2011, pp. 54-60.

[7] D. E. Paxson and J. Wilson, "Unsteady Ejector Performance: An Experimental Investigation Using a Pulsejet Driver", Paper AIAA-2002-3915, 2002.

[8] V. A. Levin, G. D. Smekhov, and A. I. Tarasov, "Designexperiment investigation of pulse detonation engine", Preprint No. 42-98, Institute of mechanics, MGU, 1998.

[9] M. G. Bulychev, "Analysis of the flapping motion feasibility study”, Polyot, No. 10, 2004.

[10] E. Yakovlev, "Blinov - Kulibin of XX century", Aircraft faculty, MAI, Krylia Rodiny, No. 8, 2005, pp. 14-17.

[11] V. I. Bogdanov, "Ways of the wing efficiency increase in pulsating flow", Polyot, No. 6, 2008, pp. 20-22.

[12] V. I. Bogdanov, "Unsteady mass interaction, inerzoids, propulsive devices with no emission of reaction mass, and the Euler law of momentum conservation", Engineer, No. 7, 2007, pp. 24-26.

[13] "History and science philosophy", Edited by Professor Yu. V. Kryanev and Professor L. E. Motorina, Study guide, M.: Alpha - M. INFRA, 2008. 
[14] G. A. Pokrovskaya, "Application of wave effects in drilling and oil-and-gas field development", Oil-and-gas well construction on land and sea, No. 1-2, 1995, pp. 30-33. 